\title{
Are Rational Expectations Equilibria with Private Information Eductively Stable?
}

\author{
Maik Heinemann \\ University of Hannover, Faculty of Economics \& Business Administration, \\ Königsworther Platz 1, D-30167 Hannover. \\ heinemann@vwl.uni-hannover.de.
}

6th February 2003

\begin{abstract}
The paper derives conditions for eductive stability of rational expectations equilibria in simple linear economic models with private information. Following Guesnerie (1992, 2002), the concept of eductive stability — based on the game-theoretical concept of rationalizability — is used. It is shown that even in a private information setting, rational expectations equilibria might be justified as a result of mental process of reasoning of the agents. The paper considers two different equilibrium concepts. First, an equilibrium, where the agents are unable to condition their forecasts on the actual market price and second, an equilibrium where this is possible. Within such a equilibrium, agents try to infer from the current market price the information of the other agents. It turns out that it is important whether agents are able to use the current market price as an additional source of information, because the conditions for eductive stability that emerge under both equilibrium concepts differ.
\end{abstract}

Key words: Rational expectations, private information, eductive stability

JEL-Classification: D 82, D 83.

\section{Introduction}

In many economic models the present values of variables depend in part on expectations of present and future values of other economic variables. In order to close such models it is therefore necessary to make an assumption that specifies how agents form their expectations. An important approach regarding the formation of expectations is the rational expectations hypothesis and because of the prevalence of this hypothesis it can surely be considered 
as a central element of modern economic theory. Nevertheless, the hypothesis of rational expectations is not without its problems. These problems are mainly related to the fundamental question how such expectations can be attained if agents do not actually begin with rational expectations. The reason is that in general additional assumptions are needed in order to verify that agents are indeed able to end up with rational expectations via evolutive or eductive learning procedures. ${ }^{1}$

A usual argument used to justify rational expectations is that it is after all in the agent's own interest to form rational expectations. While this argument seems to be convincing at first sight, it still gives no answer to the above mentioned fundamental question. It is indeed true that it is individually rational to form rational expectations if all other agents do this as well, but the same expectations are by no means individually optimal, if the other agents form nonrational expectations. Thus, in models with intrinsic uncertainty, where the variables that must be predicted itself depend on expectations, there is a coordination problem associated with the hypothesis of rational expectations. These expectations are individually rational only if it is assured that all other agents behave in the same way. We therefore need to justify, why all agents agree to form rational expectations.

As described by Guesnerie (1992), this coordination problem is equivalent to the coordination problem associated with the game-theoretical concept of Nash equilibrium. One possible way taken in the game theoretic literature to justify the Nash equilibrium concept, is to look at the rationalizability of such equilibria. Rationalizable solutions to games can be derived from two fundamental principles: Individual Bayesian rationality and common knowledge of this rationality (cf. Bernheim (1984) or Pearce (1984)). Using this concept of rationalizability in the context of rational expectations equilibria, the question thus is, whether such equilibria can be justified as a unique consequence of individual rationality and common knowledge of this rationality. If the rational expectations equilibrium of a model is indeed the unique rationalizable solution, this equilibrium can be justified as a result of an eductive learning process or mental process of reasoning on the side of the agents. In this case, following the terminology of Guesnerie (1992), the rational expectations equilibrium is called a strongly rational expectations equilibrium or an eductively stable rational expectaitons equilibrium. In what follows, the two term will be used interchangeable. Based on the classical Muth (1961) model, Guesnerie (1992) derived conditions for the rational expectations equilibrium of this model to be the unique rationalizable solution and thus being eductively stable. ${ }^{2}$ His analysis thus revealed that not every rational expectations equilibrium can be justified using the concept of rationalizability.

\footnotetext{
1 The topic of learnability of rational expectations is discussed in great detail in Evans and Honkapohja (2001).

2 Evans and Guesnerie (1993) have shown that there exists a connection between the concepts of eductive stability and expectational stability (E-stability) of rational expectations equilibria. The latter concept can be used to justify
} 
Here we extend the analysis of Guesnerie $(1992,2002)$ to the case of rational expectations equilibria with private information. We look at the case where agents are not completely informed about all aspects of the relevant model but still receive private signals that provide them with some information about these aspects. As is well known, in such models with private information the market price can aggregate this dispersed private information and therefore provides an additional source of information. Thus, in the presence of private information, two different versions of rational expectations equilibria can be distinguished. First, we might consider a rational expectations equilibrium, where agents are not allowed to condition their actions on market prices. This is the same assumption that underlies the well known cobweb model, where supply decisions are made before the actual market price becomes known. Secondly, we might consider rational expectations equilibria where agents can learn from the actual market price. In such a case it is possible for the agents to condition their actions on prices, such that they are able two use the information revealed by the market price for their own decision.

The present paper derives conditions for eductive stability of both types of equilibria and this is done with the help of a simple model. The model — based on Vives (1993) — is a model of a market for a single good with a continuum of firms. Firms face a linear demand function that is subject to stochastic shifts and the firms are privately informed about a parameter affecting their costs of production. As will be shown, the conditions for eductive stability of a rational expectations equilibrium in this model crucially depend on the underlying equilibrium concept, that is they depend on whether or not firms can learn from the actual market price. Without such learning from prices, we get a condition that is equivalent to the one derived by Guesnerie (1992) for the symmetric information case. With learning from market prices, however, we get a different condition for eductive stability of the (partially revealing) rational expectations equilibrium: The market price has to be less informative regarding the unknown cost parameter than the private signals. This condition is identical to the one derived by Heinemann (2002) in a financial market model with a finite number of traders. Moreover, it is very similar to the one derived by Desgranges et al. (1998) in the context of a private information model with finite states and signals. All in all the paper thus demonstrates that rational expectations equilibria with private information can on principle be justified as a result of eductive learning. This is possible even if agents condition their actions on current market prices. Hence, although these equilibria are quite complex, they can possibly be traced back to the two fundamental principles of individual rationality and common knowledge that form the basis of the concept

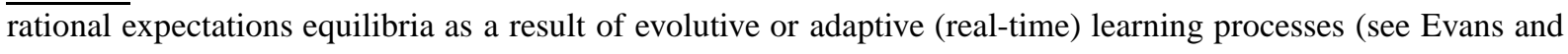
Honkapohja (1999) for a detailed survey of this subject). 
of rationalizability. However, for such a justification to be possible, specific conditions have to be fulfilled.

The remainder of the paper is organized as follows. The next Section presents the simple market model that builds the framework of the analysis. Section 3 starts with a brief overview over the concept of eductive stability using Guesnerie's original model with symmetric information. After that the conditions for eductive stability with private information and without learning from current prices are derived and discussed. Section 4 then proceeds with the analysis of the rational expectations equilibrium where the firms are allowed to learn from the current price. The paper closes with some concluding remarks.

\section{A competitive market model}

The economic model that forms the basis of the following analysis is a model of a competitive market with a continuum of risk neutral firms in the unit interval $I=[0,1]$. Market demand is random, but the inverse demand function is known to the firms:

$$
p=\beta-\frac{1}{m_{2}} x^{d}+\varepsilon
$$

Here, $x^{d}$ denotes aggregate demand and $\varepsilon$ is a normally distributed demand shock with zero mean and precision $\tau_{\varepsilon}{ }^{3} \beta>0$ and $m_{2}>0$ are known constants. The firms face increasing marginal costs that depend on an unknown parameter $\theta$. With $x(i)$ denoting the output of firm $i$, costs are $c(i)=\theta x(i)+\frac{1}{2} \frac{1}{m_{3}} x(i)^{2}$, where $m_{3}>0$. The unknown parameter $\theta$ is assumed to be normally distributed with mean $\bar{\theta}$ and precision $\tau_{\theta}$.

Every firm observes a private signal $s(i)$ that informs about $\theta$. The signal of a firm $i \in I$ is given by $s(i)=\theta+u(i)$, where $u(i)$ is normally distributed with zero mean and precision $\tau_{u}$. It is assumed that the average signal reveals the unknown value of $\theta$ by the law of large numbers, that is $\int_{0}^{1} s(i) d i=\theta$ because $\int_{0}^{1} u(i) d i=0$.

\section{Existence of an eductively stable rational expectations equilibrium without learning from prices}

\subsection{The linear rational expectations equilibrium}

In this Section we consider an equilibrium concept, where learning from current prices is impossible. This means that each firm must decide on its profit maximizing output before the

$\overline{3}$ This precision $\tau_{\varepsilon}$ is nothing more than the inverse of the variance, i.e. $\tau_{\varepsilon}=\operatorname{Var}[\varepsilon]^{-1}$ 
market price becomes known. Nevertheless, the firms will condition their production decisions on the private and public information available to them. Public information consists of the knowledge that $\theta$ is normally distributed with mean $\bar{\theta}$ and precision $\tau_{\theta}$ and private information is given by the private signals $s(i)$ for all $i \in I$. Given the above made assumptions regarding the costs of production, optimal output of a firm $i \in I$ is then given by $x^{*}(i)=m_{3} \mathrm{E}[p-\theta \mid s(i)] .^{4}$

As will be shown shortly, in a Bayesian equilibrium of the model, each firm will use a linear supply function:

$$
x^{*}(i)=m_{3}\left[\boldsymbol{\delta}(i)_{0}+\boldsymbol{\delta}(i)_{1} s(i)\right]=m_{3}\left[\boldsymbol{\delta}_{0}^{*}+\delta_{1}^{*} s(i)\right]
$$

Thus, individual supply is a linear function of the private signal $s(i)$, with coefficients $\delta_{0}^{*}$ and $\delta_{1}^{*}$ that are identical for all firms. The equilibrium coefficients $\delta_{0}^{*}$ and $\delta_{1}^{*}$ can be derived as follows. First, with $\int_{0}^{1} \delta(i)_{0} d i=\delta_{0}$ and $\int_{0}^{1} \delta(i)_{1} d i=\delta_{1}$ total supply results as $x^{s}=\int_{0}^{1} x^{*}(i) d i=m_{3}\left[\delta_{0}+\right.$ $\left.\delta_{1} \theta\right] .{ }^{5}$ With $\alpha=-m_{3} / m_{2}$, we therefore obtain from the market clearing condition $x^{s}=x^{d}$ the following equilibrium market price :

$$
p=\beta+\alpha\left[\delta_{0}+\delta_{1} \theta\right]+\varepsilon
$$

From this it follows $p-\theta=\beta+\alpha \delta_{0}+\left(\alpha \delta_{1}-1\right) \theta+\varepsilon$ and the conditional expectation of the difference $p-\theta$ of a firm $i$ with signal $s(i)$ results as: ${ }^{6}$

$$
\begin{aligned}
\mathrm{E}[p-\theta \mid s(i)] & =\beta+\alpha \delta_{0}+\left(\alpha \delta_{1}-1\right) \mathrm{E}[\theta \mid s(i)] \\
& =\beta+\alpha \delta_{0}+\left(\alpha \delta_{1}-1\right)\left[\frac{\tau_{\theta}}{\tau_{\theta}+\tau_{u}} \bar{\theta}+\frac{\tau_{u}}{\tau_{\theta}+\tau_{u}} s(i)\right] \\
& =\left(\beta+\alpha \delta_{0}+\left(\alpha \delta_{1}-1\right) \frac{\tau_{\theta}}{\tau_{\theta}+\tau_{u}} \bar{\theta}\right)+\left(\alpha \delta_{1}-1\right) \frac{\tau_{u}}{\tau_{\theta}+\tau_{u}} s(i)
\end{aligned}
$$

In an equilibrium with rational expectations, the individual coefficients $\delta(i)_{0}$ and $\delta(i)_{1}$ must therefore satisfy the following restrictions: ${ }^{7}$

$$
\delta(i)_{0}=\beta+\alpha \delta_{0}+\left(\alpha \delta_{1}-1\right) \frac{\tau_{\theta}}{\tau_{\theta}+\tau_{u}} \bar{\theta}, \quad \delta(i)_{1}=\left(\alpha \delta_{1}-1\right) \frac{\tau_{u}}{\tau_{\theta}+\tau_{u}}
$$

Integrating over all firms, these two equations allow to determine the coefficients $\delta_{0}^{*}$ and $\delta_{1}^{*}$ compatible with an equilibrium with rational expectations:

$$
\delta_{0}^{*}=\frac{1}{1-\alpha}\left[\beta-\bar{\theta} \frac{\tau_{\theta}}{\tau_{\theta}+(1-\alpha) \tau_{u}}\right], \quad \delta_{1}^{*}=-\frac{\tau_{u}}{\tau_{\theta}+(1-\alpha) \tau_{u}}
$$

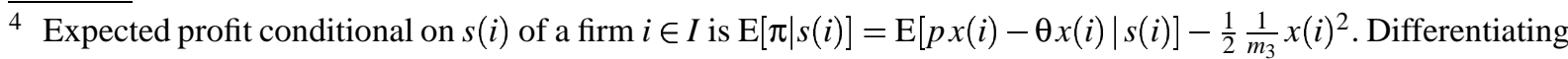
with respect to $x(i)$ yields optimal output $x^{*}(i)=m_{3} \mathrm{E}[p-\theta \mid s(i)]$.

5 The above made assumption $\int_{0}^{1} \delta(i)_{1} u(i) d i=0$ implies $\int_{0}^{1} \delta(i)_{1} s(i) d i=\delta_{1} \theta$. For details on this see Vives (1993).

6 The conditional expectation $\mathrm{E}[\theta \mid s(i)]=\frac{\tau_{\theta}}{\tau_{\theta}+\tau_{u}} \bar{\theta}+\frac{\tau_{u}}{\tau_{\theta}+\tau_{u}} s(i)$ follows from the fact that $\theta$ and $s(i)$ are normally distributed random variables (see on this DeGroot (1970)).

7 These two expression are obtained equating coefficients in equations (1) and (2).
} 


\subsection{The concept of an eductively stable rational expectations equilibrium}

Like the related game-theoretical concept of rationalizability, the concept of an eductively stable rational expectations equilibrium attempts to justify rational expectations equilibria or Nash-equilibria with the assumption of rationality. Rationalizability starts from the two fundamental hypotheses of individual rationality and common knowledge of this rationality and the model's structure. When applied to games, the question then is, which strategies will be played by rational players. As demonstrated by Guesnerie $(1992,2002)$, a related concept can be used in the context of economic models with intrinsic uncertainty where the agents have to form expectations. It might then be possible to derive the rational expectations equilibria from more basic principles, instead of merely supposing its emergence.

Starting point is the fact that under the two hypotheses stated above every firm will consider only supply quantities that maximize profits given possible rational supply quantities of other firms. In the language of game theory this means that a firm will use only those strategies that are best responses to some possible strategy profile of the other firms. Hence, strategies that are not best responses are eliminated from the strategy set of each firm. The repeated elimination of strategies that are not best responses then leads to the set of rationalizable strategies. The rational expectations equilibrium described above is necessarily an element of this set of rationalizable strategies. Following Guesnerie (1992, 2002), the rational expectations equilibrium is called a strongly rational expectations equilibrium (SREE) or eductively stable rational expectations equilibrium (ESREE), if it is the unique element of this set. In this case, it can be justified as the result of eductive learning (cf. Binmore (1987)) or mental process of reasoning on the side of the firms. ${ }^{8}$

The concept of a ESREE therefore derives a solution for the strategic problem a firm faces in case of endogenous uncertainty. The individual firm knows that the profits associated with particular supply quantities not only depend on states of nature that are beyond the firm's control. The firm also knows that its profit depends on actions that are taken by other firms. If the strategy that leads to a rational expectations equilibrium is the unique rationalizable strategy, eductive learning enables the firm to ascertain this solution given its knowledge of their opponents as well as of the economic environment.

The concept of aa ESREE can be illustrated with the help of figure 1. ${ }^{9}$ The figure is based on the classical Muth (1961) model, the same model Guesnerie (1992) used to introduce this

\footnotetext{
$\overline{8}$ Eductive learning takes place in virtual time and has to be distinguished from evolutive learning processes, where agents learn in real time and in an adaptive way from past experience (cf. (Evans and Honkapohja, 2001, p. 16f.), Guesnerie (2002)).

9 A detailed presentation of the issues underlying this simple illustration can be found in Guesnerie (1992).
} 
Figure 1. Iterative elimination of non best responses.

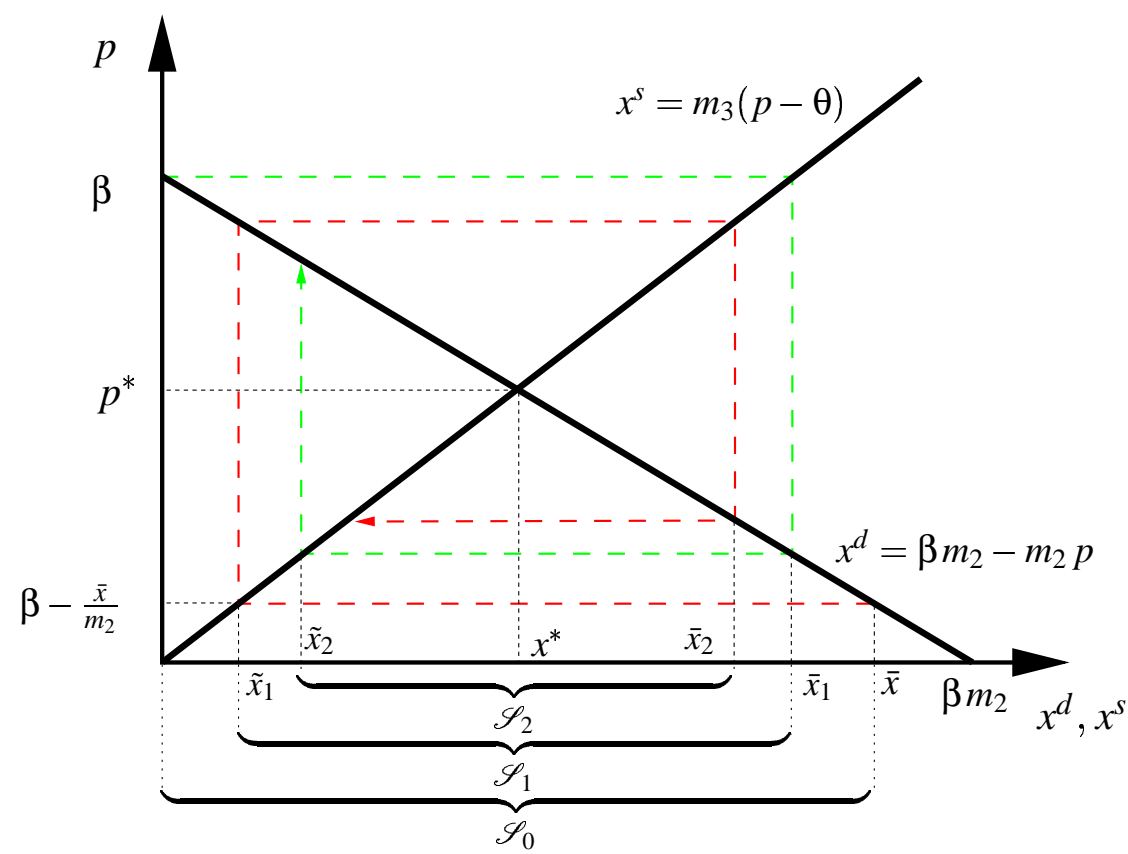

concept. For simplicity the model considered here is one without any exogenous uncertainty, i.e. market demand is deterministic and costs of production are known with certainty. Apart form this, however, it is identical to Guesnerie's model. Starting point is the assumption that the strategy set $\mathscr{S}_{0}=[0, \bar{x}]$ contains all possible supply quantities $x(i)$ of the firms. Since this is common knowledge, it is also known that market supply satisfies $x^{s} \in[0, \bar{x}]$. Regarding the market price this implies that $\beta-\bar{x} / m_{2} \leq p \leq \beta$. If this is the case, however, a profit maximizing firm never chooses supply quantities greater than $\bar{x}_{1}=m_{3}(\beta-\theta)$ or smaller than $\tilde{x}_{1}=m_{3}\left(\beta-\bar{x} / m_{2}-\theta\right)$. Therefore, after the first round of the iterative process, all supply quantities $x(i)>\bar{x}_{1}$ as well as $x(i)<\tilde{x}_{1}$ are eliminated form the firms' strategy sets. Thus, the modified strategy set $\mathscr{S}_{1}$, that results after the first round is given by $\mathscr{S}_{1}=\left[\tilde{x}_{1}, \bar{x}_{1}\right]$. Based on $\mathscr{S}_{1}$ and the fact that all this constitutes common knowledge, the same argument can be applied again and because we have $x^{s} \in\left[\tilde{x}_{1}, \bar{x}_{1}\right]$, the best replies are now characterized by the set $\mathscr{S}_{2}$ depicted in figure 1.

As can be seen from the figure, the strategy sets of the firms become smaller and smaller as the iterative process proceeds. In the present case, only the supply quantity $x^{*}$ associated with the rational expectations equilibrium survives. Obviously, the condition that must be satisfied in order to obtain such a result coincides with the well known stability condition for the cobweb dynamics. Accordingly, we must have $|\alpha|<1$ - the ratio of the slopes of the supply function and the demand function must be greater than one in absolute value - for existence of an ESREE. 


\subsection{Conditions for Existence of an ESREE}

In order to use the concept of an ESREE in the present model, some modifications relative to the analysis of Guesnerie (1992) are necessary. The reason is that in the model considered here, not only the market price $p$ is unknown as in Guesnerie's model but the cost parameter $\theta$ is unknown, too. In order to predict the market price, a firm therefore needs to form a belief how other firms react to their information regarding the unknown cost parameter $\theta$. Altogether this requires that we explicitly specify the set of strategies used by the firms. In what follows, only linear strategies are considered. ${ }^{10}$ More precisely, it is assumed that a strategy or supply decision of a firm $i \in I$ is a linear affine function of its private signal $s(i)$. Consequently, a strategy of a firm $i \in I$ is completely described by a pair $d(i)=\left(\delta(i)_{0}, \delta(i)_{1}\right)$ of real numbers and in what follows we will therefore refer to $d(i)$ as a strategy of trader $i$. The set of all strategies $\mathcal{D}(i)$ of trader $i$ is then $\mathcal{D}(i)=\left\{d(i) \mid d(i) \in \mathbb{R}^{2}\right\}$. For the individual firm only the aggregated or average behavior of the other firms matters. Therefore let $d=\left(\delta_{0}, \delta_{1}\right) \equiv$ $\int d(i) d i=\left(\int \delta(j)_{0} d j, \int \delta(j)_{1} d j\right)$ denote the average strategy resulting from a specific profile of strategies of the other firms. Moreover, let $\mathcal{D}=\left\{d=\int d(j) d j \mid d(j) \in \mathcal{D}(j)\right\}$ denote the set of all possible average strategies. Then, given an average strategy $d \in \mathcal{D}$, firm $i$ can compute aggregate supply $x^{s}=m_{3}\left[\delta_{0}+\delta_{1} \theta\right]$ as well as the difference $p-\theta=\beta+\alpha \delta_{0}+\left(\alpha \delta_{1}-1\right) \theta+\varepsilon$. Hence, the conditional expectation regarding the difference $p-\theta$ given the average strategy $d$ is:

$$
\mathrm{E}[p-\theta \mid s(i)]=\underbrace{\beta+\alpha \delta_{0}+\left(\alpha \delta_{1}-1\right) \frac{\tau_{\theta}}{\tau_{\theta}+\tau_{u}}}_{\delta^{\prime}(i)_{0}}+\underbrace{\left(\alpha \delta_{1}-1\right) \frac{\tau_{u}}{\tau_{\theta}+\tau_{u}}}_{\delta^{\prime}(i)_{1}} s(i)
$$

Equation (4) shows how a firm $i \in I$ reacts to the profile of strategies underlying the average strategy $d$ : Given $d=\left(\delta_{0}, \delta_{1}\right)$, the best response of firm $i$ is the strategy $d^{\prime}(i)=\left(\delta^{\prime}(i)_{0}, \delta^{\prime}(i)_{1}\right)$ and thus the output $x^{\prime}(i)=m_{3}\left[\delta^{\prime}(i)_{0}+\delta^{\prime}(i)_{1} s(i)\right]$. Formally, equation (4) gives rise to two equations that show how the parameters $\delta(i)_{0}$ and $\delta(i)_{1}$ of the individual strategy depend on the strategies of the other firms:

$$
\begin{aligned}
& \delta^{\prime}(i)_{0} \equiv f^{0}\left(\delta_{0}, \delta_{1}\right)=\beta+\alpha \delta_{0}+\left(\alpha \delta_{1}-1\right) \frac{\tau_{\theta}}{\tau_{\theta}+\tau_{u}} \bar{\theta} \\
& \delta^{\prime}(i)_{1} \equiv f^{1}\left(\delta_{1}\right)=\left(\alpha \delta_{1}-1\right) \frac{\tau_{u}}{\tau_{\theta}+\tau_{u}}
\end{aligned}
$$

These two equations can be used to describe the iterative process of elimination of non best responses. Since no firm uses a strategy that is not a best response, the same is true for the average strategies. Thus, based on equations (5a) and (5b) and with $d^{\prime}=\left(\delta_{0}, \delta_{1}\right)$ as well as

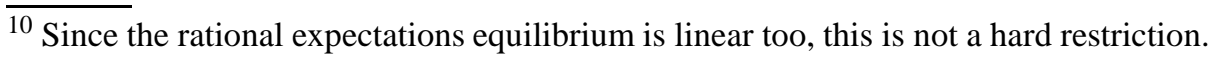


$F(d)=\left(f^{0}\left(\delta_{0}, \delta_{1}\right), f^{1}\left(\delta_{1}\right)\right)$, we may define an operator $F(d)$ that maps profiles of strategies into corresponding best responses:

$$
d^{\prime}=F(d)
$$

Obviously, the rational expectations equilibrium $d^{*}=\left(\delta_{0}^{*}, \delta_{1}^{*}\right)$ is the unique fixed point of this mapping. Moreover, because of linearity, the mapping defined by equation (6) is a contraction mapping only if this unique fixed point is stable. This in turn requires that the eigenvalues $\lambda_{1}, \lambda_{2}$ of the matrix of partial derivatives:

$$
D F(\delta)=\left(\begin{array}{cc}
\alpha & \alpha \frac{\tau_{\theta}}{\tau_{\theta}+\tau_{u}} \overline{\tau_{u}} \\
0 & \alpha \frac{\tau_{u}}{\tau_{\theta}+\tau_{u}}
\end{array}\right)
$$

have modulus less then 1 . The respective eigenvalues are $\lambda_{1}=\alpha$ and $\lambda_{2}=\alpha \frac{\tau_{u}}{\tau_{\theta}+\tau_{u}}$ and because $\alpha<0$ and $\tau_{u} \geq 0$ as well as $\tau_{\theta}>0$ we get:

Proposition 1. The rational expectations equilibrium $d^{*}=\left\{\delta_{0}^{*}, \delta_{1}^{*}\right\}$ is the unique stable fixed point of the mapping (6), if and only if $|\alpha|<1$.

Proposition 1 states a necessary condition for existence of an eductively stable rational expectations equilibrium in the simple market model given here. ${ }^{11}$ In order to justify the rational expectations equilibrium actually as a result of a eductive learning process, one additional restriction is required. The reason is that the iterative process of elimination of non best responses must start from a particular set of strategies $\mathcal{D}(i)^{0}$. Following Guesnerie (1992), it is therefore assumed that there exists a credible restriction on the set of initial strategies. In particular, it is assumed that there exists a credible restriction on the set of average strategies $\mathcal{D}$ according to which it is common knowledge that $\mathcal{D}$ is a subset $\mathcal{A}$ of the set of all linear strategies: ${ }^{12}$

Proposition 2. If there exists a credible restriction according to which $\mathcal{D} \in \mathcal{A}$, where $\mathcal{A}$ is a bounded set of linear strategies that contains the rational expectations equilibrium $d^{*}=$ $\left\{\delta_{0}^{*}, \delta_{1}^{*}\right\}$, this equilibrium is an ESREE if and only if $|\alpha|<1$.

In the present case of an equilibrium with private information and without learning from prices we therefore end up with the same condition for existence of an ESREE as in the symmetric information case that is considered by Guesnerie (1992). Figure 2 illustrates on the basis of the coefficient $\delta(i)_{1}$ of the linear strategy $d(i)$, how the iterative elimination of non best

\footnotetext{
$\overline{{ }^{11} \text { Since }} m_{3}>0$ as well as $m_{2}>0$ by assumption, we have $\alpha=-m_{3} / m 2<0$. Therefore, the above stated condition is equivalent to $-1<\alpha<0$.

${ }^{12}$ In fact, the set $\mathcal{D}$ can be choosen arbitrarily as long as it includes the REE $d^{*}$.
} 
Figure 2. Eductive learning of rational expectations without learning from prices.

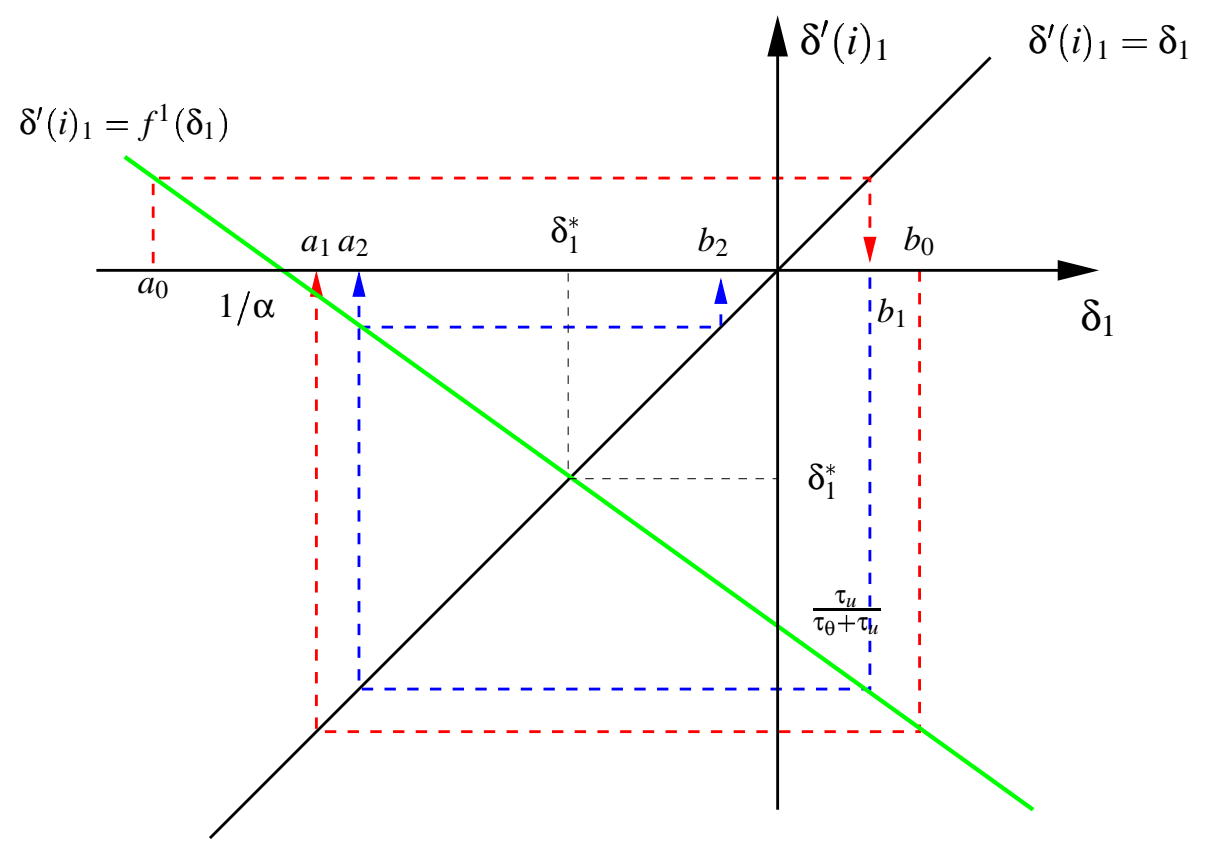

responses leads to the rational expectations equilibrium if the condition stated in proposition 2 is satisfied. ${ }^{13}$ Starting point is a credible restriction according to which $\mathcal{D} \in \mathcal{A}$. It is assumed that this restriction implies $\delta_{1} \in\left[a_{0}, b_{0}\right]$. A firm $i \in I$ therefore chooses only strategies with $\delta(i)_{1} \in\left[a_{1}, b_{1}\right]$ and so every firm can be sure that no other firm chooses strategies with an associated weight $\delta(i)_{1}$ that lies outside the interval $\left[a_{1}, b_{1}\right]$. Hence, we must have $\delta_{1} \in\left[a_{1}, b_{1}\right]$ for all average strategies. Given that this is common knowledge, the same argument can be applied again and as can be seen, this finally leads to the rational expectations equilibrium $\delta(i)_{1}=\delta_{1}^{*}$.

Despite the fact that existence of a rational expectations equilibrium is never a problem in the simple market model that is considered here, the justification of such an equilibrium is possible only if additional requirements are met. An ESREE exists only if $0>\alpha>-1$. Thus, we end up with a condition involving the ratio of the slopes of the supply function and the demand function. Moreover, this condition is identical to that derived by Guesnerie (1992) for the same model with symmetric information. The interpretation of this result is also identical with the symmetric information case. Coordination of expectations and hence existence of an eductively stable or strongly rational expectations equilibrium is unlikely, whenever it is difficult for a single firm to predict the market price, because this market price itself depends on expectations of other firms. A more detailed discussion of this issue is postponed to the end of the next section.

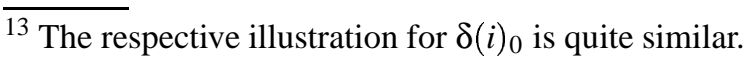




\section{Existence of an eductively stable rational expectations equilibrium with learning from prices}

\subsection{The linear rational expectations equilibrium with learning from prices}

We now look at a different equilibrium concept, where every firm is able to condition its supply decision on the actual market price. This equilibrium concept enables the firms to use the information revealed by the actual market price for their own decisions. With respect to the profit maximizing output we therefore get: ${ }^{14}$

$$
x(i)=m_{3}[p-\mathrm{E}[\theta \mid s(i), p]]
$$

The rational expectations equilibrium of this model is again linear. In equilibrium, the conditional expectation on $\theta$ given $s(i)$ and $p$ is:

$$
\mathrm{E}[\theta \mid s(i), p]=\delta(i)_{0}+\delta(i)_{1} s(i)+\delta(i)_{2} p=\delta_{0}^{*}+\delta_{1}^{*} s(i)+\delta_{2}^{*} p
$$

The equilibrium values of the coefficients $\delta_{0}^{*}, \delta_{1}^{*}$ and $\delta_{2}^{*}$ have to be determined appropriately. From equation (7) we now get the following market supply:

$$
x^{s}(p)=\int_{0}^{1} x(i) d i=m_{3}\left[\left(1-\delta_{2}^{*}\right) p-\delta_{0}^{*}-\delta_{1}^{*} \theta\right]
$$

Market clearing requires $x^{s}=x^{d}$ and with $\alpha=-m_{3} / m_{2}<0$ we end up with the following equilibrium price:

$$
p=\frac{1}{1-\alpha\left(1-\delta_{2}^{*}\right)}\left[\beta-\alpha \delta_{0}^{*}-\alpha \delta_{1}^{*} \theta+\varepsilon\right]
$$

From equation (9) and the assumptions made above, it now follows that the vector $y=$ $(\theta, s(i), p)$ is normally distributed with expectation $\mu=\mathrm{E}[y]$ and covariance matrix $\Sigma=\operatorname{Var}[y]$ :

$$
\mu=\left(\begin{array}{c}
\bar{\theta} \\
\bar{\theta} \\
\frac{\beta-\alpha \delta_{0}^{*}-\alpha \delta_{1}^{*} \bar{\theta}}{1-\alpha\left(1-\delta_{2}^{*}\right)}
\end{array}\right), \quad \Sigma=\frac{1}{\tau_{\theta}}\left(\begin{array}{ccc}
1 & 1 & \frac{-\alpha \delta_{1}^{*}}{1-\alpha\left(1-\delta_{2}^{*}\right)} \\
1 & 1+\frac{\tau_{\theta}}{\tau_{u}} & \frac{-\alpha \delta_{1}^{*}}{1-\alpha\left(1-\delta_{2}^{*}\right)} \\
\frac{-\alpha \delta_{1}^{*}}{1-\alpha\left(1-\delta_{2}^{*}\right)} & \frac{-\alpha \delta_{1}^{*}}{1-\alpha\left(1-\delta_{2}^{*}\right)} & \left(\frac{\alpha \delta_{1}^{*}}{1-\alpha\left(1-\delta_{2}\right)}\right)^{2}+\left(\frac{1}{1-\alpha\left(1-\delta_{2}^{*}\right)}\right)^{2} \frac{\tau_{\theta}}{\tau_{\varepsilon}}
\end{array}\right)
$$

Since $y \sim \mathcal{N}(\mu, \Sigma)$, the conditional variance of $\theta$ can be computed as follows: ${ }^{15}$

$$
(\operatorname{Var}[\theta \mid s(i), p])^{-1}=\tau=\tau_{\theta}+\tau_{u}+\alpha^{2} \delta_{1}^{* 2} \tau_{\varepsilon}
$$

\footnotetext{
${ }^{14}$ Expected profit of a firm $i \in I$ conditional on $s(i)$ and $p$ is given by $\mathrm{E}[\pi \mid s(i), p]=p x(i)-\mathrm{E}[\theta x(i) \mid s(i), p]-$ $\frac{1}{2} \frac{1}{m_{3}} x(i)^{2}$. Differentiation with respect to $x(i)$ yields $x(i)=m_{3}[p-\mathrm{E}[\theta \mid s(i), p]]$.

${ }^{15}$ The respective formulae for the conditional variance $\operatorname{Var}[\theta \mid s(i), p]$ and the conditional expectation $\mathrm{E}[\theta \mid s(i), p]$ are based on the fact that $\theta, s(i)$ as well as $p$ are all normally distributed (cf. DeGroot (1970)).
} 
We therefore obtain the following expression for the conditional expectation $\mathrm{E}[\theta \mid s(i), p]$ :

$$
\mathrm{E}[\theta \mid s(i), p]=\frac{1}{\tau}\left\{\beta \alpha \delta_{1}^{*} \tau_{\varepsilon}+\tau_{\theta} \bar{\theta}-\alpha^{2} \delta_{1}^{*} \delta_{0}^{*} \tau_{\varepsilon}+\tau_{u} s(i)-\left[\delta_{1}^{*} \alpha\left(1-\alpha\left(1-\delta_{2}^{*}\right)\right) \tau_{\varepsilon}\right] p\right\}
$$

In a rational expectations equilibrium, the coefficients $\delta_{0}^{*}, \delta_{1}^{*}$ and $\delta_{2}^{*}$ must satisfy the following restrictions:

$$
\delta_{0}^{*}=\frac{1}{\tau}\left[\beta \alpha \delta_{1}^{*} \tau_{\varepsilon}+\tau_{\theta} \bar{\theta}-\alpha^{2} \delta_{1}^{*} \delta_{0}^{*} \tau_{\varepsilon}\right], \quad \delta_{1}^{*}=\frac{1}{\tau} \tau_{u}, \quad \delta_{2}^{*}=-\frac{1}{\tau}\left[\delta_{1}^{*} \alpha\left(1-\alpha\left(1-\delta_{2}^{*}\right)\right) \tau_{\varepsilon}\right]
$$

The solutions are:

$$
\begin{aligned}
& \delta_{0}^{*}=\frac{\alpha \beta \tau_{u} \tau_{\varepsilon}+\tau_{\theta} \tau \bar{\theta}}{\tau^{2}+\alpha^{2} \tau_{u} \tau_{\varepsilon}} \\
& \delta_{1}^{*}=\frac{\tau_{u}}{\tau} \\
& \delta_{2}^{*}=\frac{\alpha(\alpha-1) \tau_{u} \tau_{\varepsilon}}{\tau^{2}+\alpha^{2} \tau_{u} \tau_{\varepsilon}}
\end{aligned}
$$

These three equations still contain the unknown precision $\tau$. Using equations (10) and (11b), however, we arrive at the following third order polynomial in $\delta_{1}^{*}$ :

$$
\phi\left(\delta_{1}^{*}\right)=\delta_{1}^{*}\left[\left(\delta_{1}^{*}\right)^{2} \alpha^{2} \tau_{\varepsilon}+\tau_{\theta}+\tau_{u}\right]=\tau_{u}
$$

Since the term in squared brackets on the left hand side of equation (12) is strictly positive, $\delta_{1}^{*}=0$ is the only real root of the equation $\phi\left(\delta_{1}^{*}\right)$. Moreover, we have $\phi^{\prime}\left(\delta_{1}^{*}\right)>0$, such that there exists a unique positive solution $\delta_{1}^{*}$ for equation (12). Given this solution, the remaining two coefficients $\delta_{0}^{*}$ and $\delta_{2}^{*}$ can be computed from equations (11a) and (11c).

\subsection{Conditions for existence of an eductively stable rational equilibrium with learning from prices}

As in the above considered case of an equilibrium without learning from prices, the analysis is based on linear strategies. A strategy of a firm $i \in I$ is now a linear affine function of its private signal $s(i)$ and the market price $p$. Therefore such a strategy is completely described by a triple $d(i)=\left\{\delta(i)_{0}, \delta(i)_{1}, \delta(i)_{2}\right\}$ of real numbers. ${ }^{16}$ Again $\mathcal{D}(i)=\left\{d(i) \mid d(i) \in \mathbb{R}^{3}\right\}$ denotes the strategy set of a firm $i \in I$. $d$ denotes the average strategy resulting from a particular profile of strategies of the firms and $\mathcal{D}=\left\{d=\int d(i) d i \mid d(i) \in \mathcal{D}(i)\right\}$ is the set of all those strategies.

Given any average strategy $d \in \mathcal{D}$, the conditional expectation of $\theta$ of a firm $i$ which observes the price $p$ is given by:

$$
\mathrm{E}[\theta \mid s(i), p]=\frac{1}{\tau}\left\{\beta \alpha \delta_{1} \tau_{\varepsilon}+\tau_{\theta} \bar{\theta}-\alpha^{2} \delta_{1} \delta_{0} \tau_{\varepsilon}+\tau_{u} s(i)-\left[\delta_{1} \alpha\left(1-\alpha\left(1-\delta_{2}\right)\right) \tau_{\varepsilon}\right] p\right\}
$$

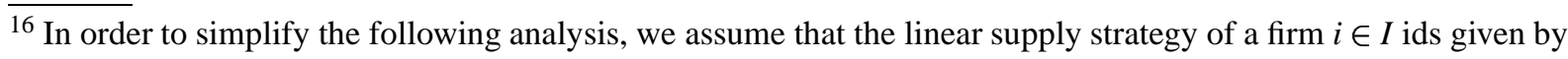
$x(i)=m_{3}\left[\delta(i)_{0}+\delta(i)_{1} s(i)+\left(\delta(i)_{2}-1\right) p\right]$.
} 
Analogous to equation (10) we have here that $\tau=\tau_{\theta}+\tau_{u}+\alpha^{2} \delta_{1}^{2} \tau_{\varepsilon}$. From equation (13) and the condition of a profit maximizing supply quantity we obtain the following three equations that specify the best response to the average strategy $d=\left\{\delta_{0}, \delta_{1}, \delta_{2}\right\}$ :

$$
\begin{aligned}
\delta^{\prime}(i)_{0} & =\frac{\beta \alpha \delta_{1} \tau_{\varepsilon}+\tau_{\theta} \bar{\theta}-\alpha^{2} \delta_{1} \delta_{0} \tau_{\varepsilon}}{\tau_{\theta}+\tau_{u}+\alpha^{2} \delta_{1}^{2} \tau_{\varepsilon}} \\
\delta^{\prime}(i)_{1} & =\frac{\tau_{u}}{\tau_{\theta}+\tau_{u}+\alpha^{2} \delta_{1}^{2} \tau_{\varepsilon}} \\
\delta^{\prime}(i)_{2} & =-\frac{\delta_{1} \alpha\left(1-\alpha\left(1-\delta_{2}\right)\right) \tau_{\varepsilon}}{\tau_{\theta}+\tau_{u}+\alpha^{2} \delta_{1}^{2} \tau_{\varepsilon}}
\end{aligned}
$$

In order to prove eductive stability we have to look at again the dynamic properties of the system of difference equations that can be derived from equations (14a) - (14c). Regarding this system, it must then be noticed that equation (14b), which specifies the coefficient $\delta(i)_{1}$ associated with the private signal $s(i)$ in the best response is now a nonlinear equation. It is, however, easily verified that this equation maps the entire real line into the closed interval $\left[0, \frac{\tau_{u}}{\tau_{\theta}+\tau_{u}}\right]$. Therefore, the analysis can be restricted to strategy sets $\mathcal{D}(i)$ for all $i \in I$ that satisfy $0 \leq \delta(i)_{1} \leq \frac{\tau_{u}}{\tau_{\theta}+\tau_{u}}$. Consider now first the difference equation which results from equation this nonlinear equation (14b):

$$
\delta_{1, t}=\frac{\tau_{u}}{\tau_{\theta}+\tau_{u}+\alpha^{2} \delta_{1, t-1}^{2} \tau_{\varepsilon}} \equiv f^{1}\left(\delta_{1, t-1}\right)
$$

The unique stationary point of this difference equation is $\delta_{1}^{*}$ and the derivative of the function $f^{1}\left(\delta_{1, t-1}\right)$ evaluated at $\delta_{1}^{*}$ is given by:

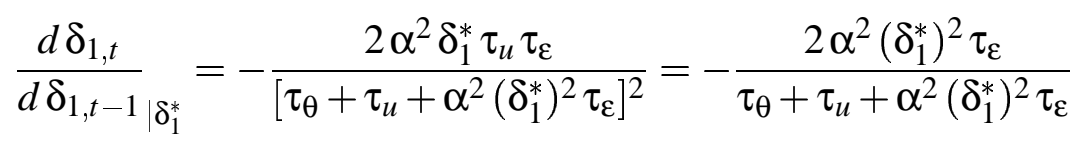

Thus, $\delta_{1}^{*}$ is a globally stable stationary point of the difference equation $\delta_{1, t}=f^{1}\left(\delta_{1, t-1}\right)$, if and only if:

$$
\alpha^{2}\left(\delta_{1}^{*}\right)^{2} \tau_{\varepsilon}<\tau_{\theta}+\tau_{u}
$$

Under the assumption that condition (15) is satisfied and provided that $\delta_{1}$ equals its respective equilibrium value $\delta_{1}^{*}$, the remaining two difference equations resulting from equations (14a) and (14c) are linear in $\delta_{0}$ and $\delta_{2}$, respectively. The respective partial derivatives of these difference equations evaluated at $\delta_{1}^{*}$ are:

$$
\frac{d \delta_{0, t}}{d \delta_{0, t-1}}=\frac{d \delta_{2, t}}{d \delta_{2, t-1}}=-\alpha^{2}\left(\delta_{1}^{*}\right)^{2} \frac{\tau_{\varepsilon}}{\tau_{u}}
$$

Therefore, the stationary points $\delta_{0}^{*}$ and $\delta_{2}^{*}$ are stable, only if:

$$
\alpha^{2}\left(\delta_{1}^{*}\right)^{2} \tau_{\varepsilon}<\tau_{u}
$$

Finally, we may note that because $\tau_{\theta}>0$, whenever condition (16) is satisfied, (15) will be satisfied too. We therefore obtain: 
Proposition 3. If there exists a credible restriction according to which $\mathcal{D} \in \mathcal{A}$, where $\mathcal{A}$ is a bounded set of linear strategies that contains the rational expectations equilibrium $d^{*}=$ $\left\{\delta_{0}^{*}, \delta_{1}^{*}, \delta_{2}^{*}\right\}$, this equilibrium is an ESREE if and only if $\alpha^{2}\left(\delta_{1}^{*}\right)^{2} \tau_{\varepsilon}<\tau_{u}$.

Since equation (12) implies that $\alpha^{2}\left(\delta_{1}^{*}\right)^{2} \tau_{\varepsilon}=\frac{\tau_{u}}{\delta_{1}^{*}}-\tau_{\theta}-\tau_{u}$, the condition stated in proposition 3 is satisfied, whenever $\delta_{1}^{*}>\frac{\tau_{u}}{\tau_{\theta}+2 \tau_{u}}$. Obviously, it depends on the parameters $\alpha, \tau_{\theta}, \tau_{u}$ as well as $\tau_{\varepsilon}$ of the model, whether or not there exists an ESREE. Its worth mentioning that this condition for existence could be satisfied even if we have $\alpha<-1$. A strongly rational expectations equilibrium with learning from prices therefore exists under weaker restrictions regarding $\alpha$ than without such learning. Indeed, the parameter $\alpha$ is important for existence of a strongly rational expectations equilibrium in the case without learning from prices only, because $\alpha$ determines how a single firm is influenced by expectations and actions of other firms. In view of a perfectly elastic demand function or an inelastic supply function, the price prediction poses no problem for the individual firm because this price is nearly unaffected by expectations (Guesnerie (1992)). With learning from prices, however, there is no problem of a price forecast at all. Since firms are able to condition their supply on the market price, the only problem is to predict the unknown parameter $\theta$. This prediction is based on the available private and public information, and the parameters that determine the informativeness of private and public signals are therefore important for existence of an ESREE. Accordingly, the condition stated in proposition 3 can be expressed in a way that accentuates the informativeness of the private signals relative to that of the market price $p$ :

Proposition 4. The condition $\alpha^{2}\left(\delta_{1}^{*}\right)^{2} \tau_{\varepsilon}<\tau_{u}$ for existence of an ESREE is equivalent to the condition that in the rational expectations equilibrium the market price $p$ is less informative regarding $\theta$ than the private signals.

PROOF: In a rational expectations equilibrium the market price $p$ is given by equation (9). From this price, the following random variable $\omega$ can be computed:

$$
\omega=\frac{1-\alpha\left(1-\delta_{2}^{*}\right)}{\alpha \delta_{1}^{*}}\left[p-\frac{1}{1-\alpha\left(1-\delta_{2}^{*}\right.}\left(\beta-\alpha \delta_{0}^{*}\right)\right]=-\theta+\frac{1}{\alpha \delta_{1}^{*}} \varepsilon
$$

Thus the market price $p$ represents a noisy signal of the unknown $\theta$. The respective informativeness (precision) of the price is given by $\tau_{\omega}=\tau_{\varepsilon}\left(\alpha \delta_{1}^{*}\right)^{2}$. By assumption, the informativeness of the private signals is given by $\tau_{u}$. Therefore, the condition stated proposition 3 is satisfied only if $\tau_{\omega}<\tau_{u}$.

Altogether, the above derived condition for existence of a strongly rational expectations equilibrium shows that the coordination of the individual expectations is difficult, if the price reveals too much information regarding the unknown parameter $\theta$. The reason for this is that a 
relatively high informativeness of the price means that the extraction of information regarding $\theta$ from the market price is quite important for the firms. However, the extraction of information from the market price requires a precise idea of the other firms' behavior, that is an idea how the other firms use their private information and the information contained in the market price $p$. The underlying problem is the well known problem of 'forecasting the forecasts of others' that is described by Keynes (1936) in his famous 'beauty contest' example. If the market price is not very informative, it is not quite important for the single firm to anticipate correctly what the other firms believe and do. Therefore, a strongly rational expectations equilibrium is likely to exist in this case.

\section{Concluding Remarks}

As the preceding sections demonstrated, it is indeed possible to justify rational expectations equilibria of a simple market model with private information and learning from current prices. If certain conditions are met, the rational expectations equilibrium can be shown to be the result of an eductive learning process, because there is only one consistent way of 'forecasting the forecasts of others'. This justification is important, because rational expectations equilibria with private information can be quite complex and a natural objection against the rational expectations hypothesis is that the required coordination of expectations seems unlikely in such a case.

Using the game-theoretical concept of rationalizability, it has been shown, that rational expectations equilibria with private information can be derived from the two fundamental principles of individual rationality and common knowledge. If the rational expectations equilibrium is the unique rationalizable solution, these two hypotheses imply that every firm will use only the unique strategy - and will thus form the unique expectation - that results in this rational expectations equilibrium. As has been shown, however, not every rational expectations equilibrium turns out to be eductively< stable, because in order for this to be the case, specific conditions have to be met.

In models with private information, the market price aggregates dispersed private information. Besides its allocative function, the price therefore fulfills an additional informational function. As has been shown, the conditions for existence of a strongly rational expectations equilibrium crucially depend on the firm's ability to use the information that prices reveal. If information revealed by prices cannot be used, because the supply decisions take place before the actual market price becomes known, the condition for existence of a strongly rational expectations equilibrium with private information coincides with the condition derived by Guesnerie (1992) for the symmetric information case. An eductively stable rational rational expectations equi- 
librium with learning from prices, however, exists only if private signals are more informative regarding the unknown cost parameter than the market price. Despite these formal differences, the conditions for existence of an eductively stable rational rational expectations equilibrium in both cases share a common feature: Existence of an eductively stable rational expectations equilibrium is more likely, when it is not quite important for the single firm to anticipate ore predict correctly what other firms believe or do. Viewed in this way, it becomes clear that the conditions derived in the preceeding analysis ensure that the problem of coordinating the firms' expectations that was mentioned in the introduction, becomes not too overwhelming. Without further restrictions it is just impossible for a firm to 'forecast the forecasts of others'. The firms therefore need reliable precognition that takes the form of restrictions on the specific characteristics of the forecasts of others. Exactly these restrictions are given by the conditions for existence of strongly rational expectations equilibria that are derived in this paper.

\section{References}

Bernheim, D., 1984, Rationalizable strategic behavior, Econometrica 52, 1007-1028.

Binmore, K., 1987, Modeling rational players, Economics and Philosophy 3, 179-214.

DeGroot, M., 1970, Optimal Statistical Decisions (McGraw-Hill, New York).

Desgranges, G., Geoffard, P. and Guesnerie, R., 1998, Educing 'revealing' equilibria, mimeo.

Evans, G. and Guesnerie, R., 1993, Rationalizability, strong rationality, and expectational stability, Games and Economic Behaviour 5, 632-646.

Evans, G. and Honkapohja, S., 1999, Learning dynamics, in: J. Taylor and M. Woodford, eds., Handbook of Macroeconomics (North-Holland, Amsterdam), 449-542.

Evans, G. and Honkapohja, S., 2001, Learning and Expectations in Macroeconomics (Princeton University Press).

Guesnerie, R., 1992, An exploration of the eductive justifications of the rational-expectations hypothesis, American Economic Review 82, 1254-1278.

Guesnerie, R., 2002, Anchoring economic predictions in common knowledge, Econometrica 70, 439-480.

Heinemann, M., 2002, Existence of strongly rational expectations equilibria on asset markets with asymmetric information, in: D. Levine, W. Zame, P. Bossaerts, Schwartz and W. Torous, eds., Proceedings of the 2002 North American Summer Meetings of the Econometric Society: Finance ((http://www.dklevine.com/proceedings/finance.htm)).

Keynes, J., 1936, The General Theory of Employment, Interest and Money (Macmillan, London). 
Muth, J., 1961, Rational expectations and the theory of price movements, Econometrica 29, $315-335$.

Pearce, D., 1984, Rationalizable strategic behavior and the problem of perfection, Econometrica $52,1029-1050$.

Vives, X., 1993, How fast do rational agents learn?, Review of Economic Studies 60, 329-347. 